\title{
STAR FORMATION PROPERTIES OF BARRED GALAXIES
}

\author{
RAFIK KANDALYAN AND ARSEN KALLOGHLYAN \\ Byurakan Astrophysical Observatory, 378433 Byurakan, Armenia \\ E-mail: rkandali@bao.sci.am \\ rafik@ipia.sci.am
}

\section{Abstract}

The main results of this study can be summarized in the following:

(a) Magnitude-limited complete sample of barred galaxies was constructed. Space density of barred and unbarred galaxies at low luminosity range ( $M>$ 20 ) almost the same. At the high $(\mathrm{M}<-20)$ luminosity range about $30 \%$ of the galaxies are barred ones.

(b) Bars do not have obvious influence on active nuclei. Presence or absence of bars does not related with the large scale galaxy environment.

(c) Barred spirals, on average, have significantly higher relative star formation rate than that do unbarred galaxies.

(d) Barred spirals exhibit tight correlations between FIR, radio and X-ray luminosities, while for unbarred spirals these luminosities do not correlated, which indicates on enhanced star formation activity in barred galaxies.

(f) Group and non-group barred galaxies may differ by star formation activity.

\section{Results}

We have selected from LEDA database all barred galaxies which are north of declination - 10 degree. Both $\log N-m$ and $V / V_{m}$ tests showed on completness limit of 13.5 magnitude for the sample of barred galaxies. Additional data have been extracted from NED database. The same manner was used in construction of unbarred galaxies only they were selected from declination range $[0,+30]$. According to our data barred and unbarred galaxies do not differ neither by presence of AGN (see, also Ho et al., 1996) nor by group member. Space density of barred and unbarred galaxies at low lu- 
minosity range $(M>-20)$ almost the same. At the high $(M<-20)$ luminosity range about $30 \%$ of the galaxies are barred ones.

To verify the similarity or difference between two samples we compare cumulative distributions of star formation indicators (generally $L_{f i r} / L_{b}$, $f 12 / f 25, f 60 / f 100$, radio continuun etc). We find that barred galaxies show enhanced star formation activity with respect to unbarred objects for all morphological types. According to our analysis there are tight relations between FIR, radio and X-ray emission for our sample of barred galaxies. When we have investigated same relations for unbarred galaxies we find that these quantities are not correlated.

Now we shall discuss star formation properties of barred galaxies of different morphological types. Our analysis shows that among barred galaxies intermediate type spirals are more active in star formation than early type. We find that group barred galaxies do not stand out in star formation properties from the non-group objects when infrared, radio continuum and X-ray global parameters are considered as indicators of star formation activity. However there are significant differences in U-B and B-V colours between group and non-group barred galaxies. Namely, group barred galaxies are shown redder colours than non-group objects, while for group and nongroup unbarred galaxies we dont see such differences. It is possible that this difference in colours between group and non-group barred galaxies is due to by star formation activity.

Unlike Huang et al. (1996) we do not obtained any difference between HI content of barred and unbarred objetcs. This may indicates that atomic gas does not directly connected with star formation since star formation occurs in molecular gas.

\section{Acknowledgements}

R.K. is grateful to IAU for providing travel grant to attend IAU GA. This research has made use of the Lyon-Meudon Extragalactic Database (LEDA), suplied by the LEDA team at CRAL-Observatoire de Lyon (France) and the NASA-IPAC Extragalactic Database (NED) which is operated by the Jet Propulsion Laboratory, Caltech, under contract with the National Aeronautics and Space Administration (USA).

\section{References}

Ho L.C. et al., (1996) The effect of bars on the fueling of star formation and nonstellar activity in Galaxy nuclei, in: Barred Galaxies, ASP Gonf. Ser., Vol. 91, pp. 188-196, Buta R., Crocker D.A., Elmegreen B.G. (eds)

Huang J.H. et al., (1996) The bar-enhanced star-formation activities in spiral galaxies, $A \& S$, Vol. 313, pp. 13-24 